\title{
Development of varieties of spring bread wheat using DH lines in Western Siberia
}

\author{
Belan I.A. ${ }^{1 *}$, Rosseeva L.P. ${ }^{1}$, Blokhina N.P. ${ }^{1}$, Mukhina Y.V. ${ }^{1}$, Trubacheeva N.V. ${ }^{2}$, \\ Pershina L.A. ${ }^{2}$ \\ ${ }^{1}$ Omsk Agricultural Scientific Center, Omsk, Russia \\ ${ }^{2}$ Institute of Cytology and Genetics, SB RAS, Novosibirsk, Russia \\ * email: belan_skg@mail.ru
}

Climate changes, the impact of man-made and anthropogenic factors lead to changes in the growing conditions of crops, which requires the development of high-quality and productive varieties that are adaptive to the growing conditions. As the initial forms for breeding, it is important to use introgression lines, which are carriers of genes that control agronomically valuable traitsWhen using the conventional approach, to stabilize the exhibition of target traits in introgression lines, it is necessary to obtain at least eight self-pollinated generations. So, in bread wheat, it takes from 12 to 15 years to develop a variety using conventional breeding approach. In the laboratory of spring bread wheat of the Omsk Agricultural Scientific Center for the development of varieties of spring bread wheat, along with conventional breeding approach, the use of homozygous DH lines was tested both to accelerate the breeding process and to create promising hybrid combinations with complexes of agronomically valuable traits. Crossing aline DH-17 derived from the alloplasmic recombinant line (H. vulgare) - T. aestivum with alineCom 37-1RS.1BL (CIMMYT) resulted in a hybrid population 311-00-00. Three lines, as a result of breeding trials in different regions, became varieties of spring bread wheat included in the State Register of Breeding Achievements approved for use: Sigma and Uralosibirskaya 2, and the variety Ishimskaya 11 is protected by a patent. The variety Sigma was recommended for cultivation in the 10th regions of the Russian Federation; the variety Uralosibirskaya 2 was recommended in the 9th and 10th regions of the Russian Federation, and from 2021 in the Republic of Kazakhstan.In the Omsk region, the variety Sigma occupies about 50 thousand hectares. The fourth line, was included in the pedigree of the variety Sakmara, which is undergoing State Variety Trial in the Russian Federation. Thus, the four varieties of spring bread wheat have beendeveloped with the participation of the alloplasmic lineDH-17.Another approach was usedwhen including in breeding alloplasmic introgression DH lines DH-48-3 and DH-48-18, which combine resistance genes to powdery mildew, leaf rust, and stem rust. Since 2019 in the nursery of competitive variety trial(CVT). In terms of yield, the lines DH 48-3 and DH 48-18 on average for two years exceeded the standard variety PamyatiAzieva (2.58 t/ha) by more than $3 \mathrm{t} / \mathrm{ha}$, with yields of 6.02 and $6.31 \mathrm{t} / \mathrm{ha}$ respectively. Thus, based on the technology of accelerated breeding using DH lines, it took 6 years to develop the variety. In parallel, using the conventional breeding approaches over the years varieties of spring bread wheat Omskaya 42, Omskaya 44, Omskaya 45 and OmskayaKrepost have been released, each of which took over 10 years to be developed.

Acknowledgments: Some aspects of this work were supported by the RFBR grant No. 20-016-00196. 